\title{
System theory and sustainable development
}

\section{Opinion}

Chemical engineering as a very rich discipline by its nature has witnessed considerable expansion in the last three decades. The most successful departments in the USA and Canada as well as most of the rest of the world have been those that expanded in the direction of becoming Chemical and Biological Engineering (CBE) Departments; (e.g.: University of Colorado, Boulder, Colorado, USA; University of British Columbia, Vancouver, Canada; Iowa State University, Ames, Iowa, USA; University of Alabama, Tuscaloosa, Alabama, USA, Department of Chemical and Biological Engineering, Rensselaer Polytechnic Institute, Troy, New York, USA; Department of Chemical and Biological Engineering, Tufts University, Medford, MA, USA, etc.) with biological engineering meaning biochemical and biomedical engineering. Very similar names are, of course, also included in this category (e.g.: Division of Chemical and Bimolecular Engineering, School of Chemical and Biomedical Engineering, Nan yang Technological University, Singapore; Chemical and Bimolecular Engineering Department, University of Houston, Houston, Texas, USA; Chemical and Bimolecular Engineering Department, University of Notre Dame, Notre Dame, IN,USA, etc.).

Other departments went half the way and became departments of Chemical and Bio-Chemical Engineering only (e.g.: Department of Chemical and Biochemical Engineering, Rutgers University, Piscataway, NJ, USA; Department of Chemical and Biochemical Engineering, University of Maryland Baltimore County, Baltimore, MD,USA; Department of Chemical and Biochemical Engineering, The University of Western Ontario, London, Ontario, Canada, Chemical and Biochemical Engineering Department, Institute Tecnolo' gico de Veracruz, Veracruz, Ver., Mexico; etc.) and all these departments did reasonably well. Some Chemical and Biochemical Engineering Departments split into two separate departments (e.g.: University College, London University, London, UK) and both are doing reasonably well. Other departments became departments of Chemical and Materials Engineering (CME) (e.g.: Department of Chemical Engineering and Materials Science, Michigan State University, East Lansing, Michigan, USA, etc) and others became Chemical and Environmental Engineering (CEE) Departments (e.g. The Department of Chemical and Environmental Engineering, University of Arizona, Tucson, AZ, USA, etc.) and did also reasonably well. It will be a great success to have all 4 disciplines (Chemical, Biological, Materials and Environmental Engineering) in one department and also to include the new comers discussed later and using a new approach suitable for such a Cross-Disciplinary (CD) rich new specializations. The 4 components are all Engineering Disciplines (EDs) and using the new approach will make them fall well under the same umbrella. However the $\mathrm{CD}$ approach extends to include not only EDs but also scientific disciplines such as chemistry, physics, mathematics, microbiology, etc. as well other disciplines such as economics, politics, sociology, etc

This in addition to the new comers discussed below will make the graduates truly ready for $\mathrm{CD}$ jobs in industry as well as for research work in both academia and industry as well as governmental research centers. It is also important to emphasis the fact that the new engineering era requires that graduates be prepared not only for design

\author{
Volume I Issue I - 2017
}

\author{
Said S Elnashaie \\ Department of Chemical and Biological Engineering, University \\ of British Columbia, Canada
}

Correspondence: Said S Elnashaie, Department of Chemical and Biological Engineering, College of Engineering, University of British Columbia,Vancouver, Canada,

Email sselnashaie@gmail.com

Received: July 18, 2017 | Published: July 20, 2017

and operation, but also for innovation and should not be taught to learn only while in school and university but also to be taught the culture, ideology and philosophy of Long Life Learning (L3 according to the ABET terminology). With all this branching, interaction and synergy between disciplines and these new requirements of modern industry and the healthy forms of globalization a new approach based on ST and may be called ISA should be adopted both in under- graduate and post-graduate teaching and research in academia and will naturally spread to industry and governmental research centers.

The 4 disciplines mentioned above are relatively well defined and discussed in the literature. This does not mean that the last word has been said with regard to these disciplines, but it means that they are matured enough to be considered as given in a paper like this introducing some, may be, new and useful principles to this continuously expanding and complicating field. The first question now is: who are the new comers, and are they really new comers or intelligent extension of existing disciplines? These relatively new comers are: Sustainable Development Engineering, (SDE) which is a subsystem of Sustainable Development (SD) as a whole system. In terms of ST and the ISA, we can consider SD itself as a system formed of technical and non-technical subsystems and SDE is a subsystem of the technical subsystem as shown in some details later. This relatively new comer is an extension of Environmental Engineering (EE) which is itself an extension of Efficient Engineering (EE' the dash is to distinguish it from EE). In other words if SDE (which is a subsystem of the technical subsystem of SD) is a system then EE is a subsystem of it and EE is a subsystem of EE'. While in the terms of stability theorem EE' can be considered necessary but not sufficient for EE, what makes it sufficient is the introduction of Environmental Regulations(ERs) which came into the picture in the last 4 decades only in some countries while other countries did not introduce it in a proper manner affecting negatively the rest of the world. Similarly $\mathrm{EE}$ is necessary but not sufficient for SDE (which is itself necessary but not sufficient for SD) what makes it sufficient is the introduction of Renewable Raw Materials (RRMs) to replace the Non-RRMs. This brings into the picture not only Bio-Fuels (BFs) from wastes and energy crops (and not from food) and micro-algae, but also IntegratedBio-Refineries (IBRs) since societies do not live only on fuels. This will be made clearer later. Figure 1 shows a diagram for SD and its subsystems. 


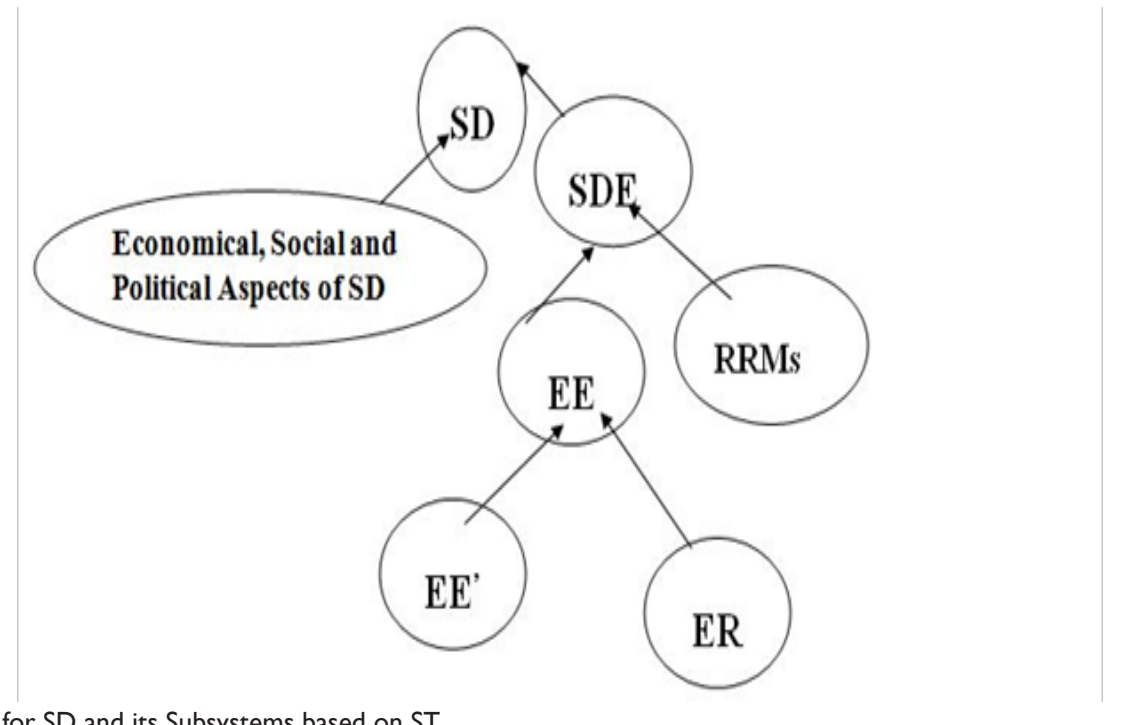

Figure I Simplified Diagram for SD and its Subsystems based on ST. Acknowledgements

None.

\section{Conflict of interest}

Author declares that there is no conflict of interest. 